\section{Investigación descriptiva, correlacional o cualitativa}

Pensar en Movimiento:

Revista de Ciencias del Ejercicio y la Salud

ISSN 1659-4436

Vol. 19, No.2, pp. 1- 17

Abre $1^{\circ}$ de julio y cierra 31 de diciembre de 2021

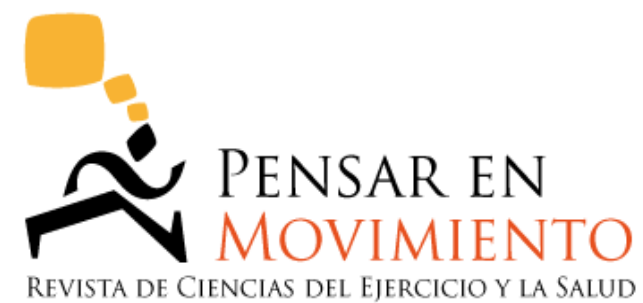

REVISTA DE CIENCIAS DEL EJERCICIO Y LA SALUD

\title{
FUENTES DE CONOCIMIENTO DE LOS ENTRENADORES: ESTUDIO DE CASO DE LAS SELECCIONES BRASILEÑAS DE BALONMANO MASCULINO ${ }^{1}$
}

\section{FONTES DE CONHECIMENTOS DOS TREINADORES: ESTUDO DE CASO DAS SELEÇÕES BRASILEIRAS MASCULINAS DE HANDEBOL}

\section{COACHES' KNOWLEDGE SOURCES: A CASE STUDY OF BRAZILIAN MEN'S HANDBALL TEAMS}

\author{
José Carlos Mendes, Ph.D (iD 1(B-C-D-E), Michel Milistetd (iD) 2(B,E), \\ Sergio José Ibáñez, Ph.D (D) 3(D-E) y Juarez Vieira do Nascimento, Ph.D (D) 2(B-C-E) \\ spock12hand@hotmail.com; michel canhoto@hotmail.com; sibanez@uex.es; \\ juarez.nascimento@ufsc.br \\ 1 Universidad Estatal del Oeste de Paraná, Paraná, Brasil \\ 2 Universidad Federal de Santa Catarina, Florianópolis Brasil \\ ${ }^{3}$ Universidad de Extremadura, Extremadura, España
}

Envío original: 2020-03-17 Reenviado: 2020-10-02

Aceptado: 2020-10-21 Publicado en versión en español: 2021-07-01

Doi: https://doi.org/10.15517/pensarmov.v19i2.47575

\section{RESUMEN}

Mendes, J. C., Milistetd, M., Ibáñez, S.J. y Vieira do Nascimento, J. (2020). Fuentes de conocimiento de los entrenadores: estudio de caso de las selecciones brasileñas de

\footnotetext{
1 Traducción al español. Original en portugués disponible en: Mendes, J., Milistetd, M., Ibáñez, S., e Nascimento, J. (2020). Fontes de conhecimentos dos treinadores: estudo de caso das seleções brasileiras masculinas de handebol. Pensar en Movimiento: Revista de Ciencias del Ejercicio y la Salud, 1(2), e41103. doi: https://doi.org/10.15517/pensarmov.v18i2.41103
} 
balonmano masculino. Pensar en Movimiento: Revista de Ciencias del Ejercicio y la Salud, 19(2), 1-17. El estudio ha identificado las fuentes de conocimiento más valorizadas por los entrenadores y ayudantes de campo de las selecciones de balonmano masculino de Brasil en las categorías juvenil (U19), júnior (U21) y adulta. La investigación trata de un estudio de caso realizado con tres entrenadores y tres entrenadores asistentes de las selecciones masculinas de balonmano de Brasil. Se recolectaron los datos por medio del cuestionario de Perfil de Formación del Entrenador. Los datos fueron analizados por medio de estadística descriptiva (mediana) e inferencial ( $U$ de Mann Whitney y Kruskal Wallis) con nivel de significancia de $p \leq .05$. Los resultados indicaron que, tanto los entrenadores como los ayudantes de campo de las selecciones, asignaban mayor valorización a las fuentes de conocimiento procedentes de "experiencia profesional" y de "formación académica". Además, se observó que las fuentes de conocimiento procedentes de "experiencia profesional" fueron más valorizadas por los integrantes de las comisiones técnicas de las selecciones U19 y adulta, mientras que los miembros de la comisión técnica de la selección U21 valorizaban más las fuentes de conocimiento procedentes de "formación académica". No obstante, no se encontraron diferencias significativas en los resultados entre entrenadores y entrenadores asistentes, así como entre las comisiones técnicas de las selecciones U19, U21 y adulta. La actuación profesional de los entrenadores y asistentes de campo de las selecciones brasileñas masculinas de las categorías $U 19, \mathrm{U} 21$ y adulta de balonmano y la construcción del proceso de entrenamiento del modelo de juego propuesto para las selecciones se estructuraron a partir de las fuentes de conocimientos procedentes de su "experiencia profesional" y la "formación académica".

Palabras clave: balonmano, entrenador, fuentes de conocimiento, Brasil.

\section{RESUMO}

Mendes, J.C., Milistetd, M., Ibáñez, S.J. e Vieira do Nascimento, J. (2020). Fontes de conhecimentos dos treinadores: estudo de caso das seleções brasileiras masculinas de handebol. Pensar en Movimiento: Revista de Ciencias del Ejercicio y la Salud, 19(2), 117. O estudo identificou as fontes de conhecimentos mais valorizadas pelos treinadores e treinadores assistentes das seleções de handebol masculino do Brasil nas categorias juvenil (U19), júnior (U21) e adulta. A pesquisa trata-se de um estudo de caso realizado com três treinadores e três treinadores assistentes das seleções masculinas de handebol do Brasil, sendo os dados coletados por meio do questionário de Perfil de Formação do Treinador. Os dados foram analisados por meio de estatística descritiva (mediana) e inferencial (U de Mann Whitney e Kruskal Wallis) com nível de significância de $p \leq .05$. Os resultados indicaram que, tanto os treinadores, como os treinadores assistentes das seleções, atribuíam maior valorização das fontes de conhecimentos procedentes da "experiência profissional" e da "formação acadêmica". Além disso, observou-se que as fontes de conhecimento procedentes da "experiência profissional" foram mais valorizadas pelos integrantes das comissões técnicas das seleções U19 e adulta, enquanto os membros da comissão técnica da seleção U21

$-2$

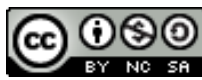

Esta obra está bajo una 
valorizavam mais as fontes de conhecimento procedentes da "formação acadêmica". Contudo não foram encontradas diferenças significativas nos resultados entre treinadores e treinadores assistentes, como também entre as comissões técnicas das seleções U19, U21 e adulta. A atuação profissional dos treinadores e treinadores assistentes das seleções brasileiras masculinas das categorias U19, U21 e adulta do Handebol e a construção do processo de treino do modelo de jogo proposto para as seleções estruturam-se a partir das fontes de conhecimentos procedentes da sua "experiência profissional" e da "formação acadêmica".

Palavras-chave: handebol, treinador, fontes de conhecimento, Brasil.

\begin{abstract}
Mendes, J.C., Milistetd, M., Ibáñez, S.J. \& Vieira do Nascimento, J. (2020). Coaches' knowledge sources: a case study of Brazilian men's handball teams. PenSAR EN Movimiento: Revista de Ciencias del Ejercicio y la Salud, 19(2), 1-17. The aim of the current study is to identify the knowledge sources most valued by coaches and assistant coaches of male Brazilian handball teams in the youth (U19), junior (U21), and adult categories. The case study included three coaches and three assistant coaches of male Brazilian handball teams. Data was collected using the Coach Training Profile Questionnaire and analyzed using descriptive (median) and inferential statistics (Mann-Whitney $U$ and Kruskal-Wallis tests), at $p \leq .05$ significance level. Results indicated that coaches and assistant coaches of the national handball teams give greater value to knowledge sources under "professional experience" and "academic training". In addition, "professional experience" was the knowledge source most valued by members of the technical commission of the U19 and adult categories, whereas members of the technical committee of the U21 category most valued "academic training" as their knowledge source. However, no significant differences were observed in results between coaches and assistant coaches, as well as between the technical commissions of the U19, $\mathrm{U} 21$, and adult categories. The professional performance of coaches and assistant coaches of Brazilian male handball teams belonging to the U19, U21, and adult categories and the construction of the training process associated with the match model proposed for the teams are structured based on knowledge sources such as "professional experience" and "academic training".
\end{abstract}

Keywords: handball, coach, knowledge sources, Brazil.

El balonmano, práctica deportiva competitiva de "organización compleja, no lineal, dinámica y auto-organizada" (Oliver, 2018, p. 229), ocurre en un ambiente con elevado nivel de incertidumbre, exigiendo toma de decisiones de alta complejidad, en función de las características individuales de los jugadores, las condiciones de la tarea a ser resuelta y las características de los adversarios (Araújo, Teques, Hernández-Mendo, Reigal, y Anguera, 2016; Travassos, Davids, Araújo, y Esteves, 2013). Un proceso de entrenamiento efectivo para tales acciones exige tareas de entreno capaces de replicar las dinámicas y los esfuerzos

$-3-$

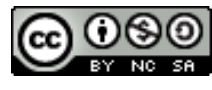

Esta obra está bajo una 
específicos con mayor incidencia durante el juego (Clemente, Martins, y Mendes, 2014; HillHaas, Dawson, Impellizzeri, y Coutts, 2011), asociadas con adecuada estructuración y jerarquización de objetivos, distribución de los contenidos y la progresión de las tareas (Cañadas, Rodríguez, Feu, Parejo, y García, 2013; Feu, 2006; Ibáñez, 2008; Tarodo, Belmonte, Toro, y Ruano, 2011).

Los entrenadores desempeñan un papel tan fundamental para la efectividad en el proceso de entrenamiento (Ibáñez, Mendoza, y Calvarro, 1999), que estudios con entrenadores deportivos se tornaron una de las tendencias emergentes en el área de las Ciencias del Deporte (Borms, 2008) y se observa un crecimiento de las publicaciones en los distintos países (Cushion et al., 2010; Galatti et al., 2016; Ibáñez, Pérez-Goye, García-Rubio, y Courel-Ibáñez, 2019). Además, el International Council for Coaches' Excellence (ICCE), organización internacional dedicada al desarrollo de entrenadores, implementa acciones en el establecimiento de fuertes alianzas entre instituciones de enseñanza, federaciones deportivas y organizaciones nacionales de deporte, con el objetivo de sumar esfuerzos comunes para mejorar la formación y el desarrollo profesional de los entrenadores en distintos niveles competitivos en el mundo (ICCE, 2013).

A pesar de no existir un modelo común de formación de entrenadores en el mundo, la habilitación de la profesión de entrenador deportivo puede alcanzarse por dos vías: la primera, basada en cursos de educación superior en las áreas de Educación Física y/o Ciencias del Deporte $y$, la segunda, en cursos promovidos por las federaciones de distintas modalidades deportivas para diferentes niveles competitivos de desempeño del entrenado (Falcão, Bennie, y Bloom, 2015; Feu, 2018). Por otro lado, existe una destacada línea de investigación que indica un consenso para el desarrollo profesional de los entrenadores por medio de distintas formas de educación formal, no formal e informal (Jiménez, Lorenzo, y Gómez, 2009; Lyle y Cushion, 2010; Nelson, Cushion, y Potrac, 2006; Trudel y Gilbert, 2006; Werthner y Trudel, 2006; Wright, Trudel, y Culver, 2007), lo que evidencia la valorización de fuentes de conocimiento procedentes de las experiencias de la práctica profesional, de las experiencias en las comunidades de práctica y carrera deportiva, como también el acceso a literatura específica y cursos de formación especializada (Cunha, Estriga, y Batista, 2014; GonzálezRivera, Campos-Izquierdo, Villalba, y Hall, 2017; Jiménez, Lorenzo, y Gómez, 2009).

En Brasil, las investigaciones son escasas e incipientes y no presentan una agenda clara de investigación; por ejemplo, una revisión sistemática realizada en las revistas que cubren el período de 2000 a 2015 constata una media de cinco artículos publicados por año, de los cuales la mayor parte estaba enfocada en aspectos relacionados a ideas de los entrenadores sobre distintos temas y en sus comportamientos (Galatti et al., 2016). En los estudios localizados sobre los aspectos formativos y desarrollo profesional de los entrenadores, los resultados enfatizan una amplia valorización de las fuentes de conocimiento procedentes de los cursos de grado en Educación Física, con énfasis en los conocimientos técnicos y en los conocimientos procedentes de las Ciencias del Deporte (Ramos, Graça, Nascimento, y Silva, 2011; Rodrigues, Teixeira Costa, Santos Junior, y Milistetd, 2017). Además, los estudios exclusivos con entrenadores de Balonmano se basaron en las ideas de los entrenadores sobre el proceso enseñanza-aprendizaje de esta modalidad deportiva en distintas categorías de formación o en la comprensión del desarrollo táctico-técnico del juego

$-4-$

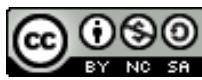

Esta obra está bajo una 
(Menezes, 2018; Menezes, Marques, y Nunomura, 2017; Menezes, Ramos, Marques, y Nunomura, 2018; Menezes, y Reis, 2014).

Las selecciones brasileñas de balonmano han alcanzado elevados niveles competitivos en la última década, como, por ejemplo, el título de campeón mundial de la selección femenina adulta en Servia en el 2013 y el reciente noveno lugar en el Campeonato Mundial masculino de 2019. Asociado con la evolución de los resultados, se observaron inversiones en centros de entrenamiento, migración de atletas para las principales ligas de Balonmano de Europa (Asobal, Bundesliga, entre otras), además de las inversiones financieras en la preparación de las selecciones para los Juegos Olímpicos Río 2016. Sin embargo, un factor relevante de los resultados alcanzados se refleja en la contratación de entrenadores extranjeros para dirigir las selecciones adultas, como también estructurar el proceso de entrenamiento de las selecciones en las etapas formativas (cadete, juvenil y junior).

En el caso de las selecciones masculinas, este proceso se materializó de forma concreta, debido a que el entrenador extranjero creó campamentos para el desarrollo técnicotáctico de los jugadores y entrenadores, en los cuales fue implementada una concepción y filosofía de entrenamiento para el desarrollo de esta modalidad deportiva y para atender las demandas del modelo de juego de la selección adulta (Amorim, 2017). A pesar de innumerables campamentos realizados en el ciclo de preparación olímpica de las selecciones brasileñas (2013-2016), no se detectaron estudios sobre el proceso de formación y desarrollo de estos entrenadores, lo que demostró un escaso interés de la comunidad científica. Sin embargo, en otros países hay una preocupación constante sobre el proceso de formación y desarrollo de estos entrenadores deportivos (Feu, 2018), por ejemplo, sobre las fuentes de conocimiento más significativas para los entrenadores en el ejercicio profesional y en la estructuración del proceso de entrenamiento del modelo de juego de las selecciones masculinas (Cunha et al., 2014; Feu, Ibáñez, Calvo, Saiz, y Cañadas, 2012; González-Rivera et al., 2017).

Dado lo anteriormente expuesto, el estudio buscó identificar las fuentes de conocimiento (FC) más valorizadas por los entrenadores y entrenadores asistentes en el ejercicio profesional y en la construcción del proceso de entrenamiento de las selecciones masculinas de balonmano en las categorías juvenil (U19), júnior (U21) y adulta.

\section{MATERIALES Y MÉTODOS}

\section{Participantes}

El estudio fue clasificado como estudio de caso (Montero y León, 2007) con la participación voluntaria de tres entrenadores y tres entrenadores asistentes de las selecciones brasileñas masculinas de balonmano de las categorás juvenil (U19), junior (U21) y adulta. Los participantes tenían una edad media de $48.66 \pm 7.50$ (35 - 54) años de edad, con grado y posgrado en Educación Física, excepto el entrenador de la selección adulta, con formación exclusiva de entrenador de balonmano certificado por la Asociación de Entrenadores de Balonmano (AEBM) de España. Los participantes tenían más de 10 años de experiencia como entrenadores en niveles competitivos nacionales e internacionales. Los entrenadores tenían en promedio $22.66( \pm 5.11)$ años de experiencia profesional en el balonmano de alto nivel, mientras que los entrenadores asistentes tenían una media de $17.66( \pm 6.22)$ años; la comisión técnica de la selección U21 acumulaba el menor tiempo de experiencia profesional en el

$-5-$

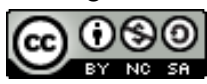

Esta obra está bajo una 
balonmano de alto nivel. Además, vale destacar que el entrenador de la selección U21 y el entrenador asistente de la selección U19 fueron atletas profesionales de esta modalidad deportiva con participación en Campeonatos Mundiales y Juegos Olímpicos (Barcelona 1992, Atlanta-1996, Atenas-2004 y Pekín - 2008).

\section{Instrumento para la recolección de datos}

Para la recolección de datos se utilizó el Cuestionario de perfil de Formación del Entrenador (QPFT, por sus siglas en portugués), originalmente propuesto por Feu et al. (2012) en idioma español, traducido y adaptado al escenario brasileño por Mendes et al. (2019), que establece las procedencias de las fuentes de conocimiento de los entrenadores en tres dimensiones: Formación Académica ( $F A)$, Experiencia de Atleta (EA) y la Experiencia Profesional (EP). Cada dimensión posee cinco indicadores que contemplan los momentos de aprendizajes, experiencias, conocimientos, metodologías y ejercicios vivenciados por los entrenadores (Tabla 1). El instrumento está compuesto de 15 preguntas con respuestas en escala tipo Likert de 1 a 10 ( 1 = Completamente en Desacuerdo y $10=$ Completamente de Acuerdo). Además, se adaptó la pregunta original del instrumento, la cual comprende: “¿Para mi desempeño profesional en la construcción de las tareas del proceso de entreno de las selecciones, como entrenador yo prefiero?". 
Tabla 1

Dimensiones e indicadores de la versión brasileña de la escala de las fuentes de conocimiento de los entrenadores

\begin{tabular}{l|l}
\hline Dimensiones & $\begin{array}{l}\text { ¿Para mi desempeño profesional y en la construcción de las tareas del proceso } \\
\text { de entrenó de las selecciones, como entrenador yo prefiero? }\end{array}$ \\
\hline \multirow{2}{*}{ Experiencia } & I) Las experiencias que adquirí a lo largo de mi carrera como atleta. \\
Atleta & II) Los conocimientos adquiridos de los entrenadores que me entrenaron. \\
III) Métodos que eran utilizados por mis entrenadores. \\
IV) Lo que aprendí con otros entrenadores mientras era atleta. \\
V) Los ejercicios y tareas que aprendí cuando era atleta.
\end{tabular}
Fuente: elaborado por los autores, 2020.

\section{Procedimientos para la recolección de datos}

Para la aplicación de la versión brasileña del QPFT, el estudio fue presentado y aprobado (Parecer $\mathrm{n}^{0} 1835025$ ) por el Comité de Ética en Investigación con Seres Humanos de la Universidad Estatal del Oeste de Paraná (UNIOESTE). Los entrenadores y entrenadores asistentes fueron invitados durante las etapas de entrenamiento de sus respectivas selecciones en las temporadas deportivas de 2016 y 2017. Posteriormente, los cuestionarios fueron enviados por medio de correo electrónico con una opción de llenar el documento en formato Word Office 2003 o vía formulario de Google Docs.

\section{Análisis de datos}

La distribución de los datos mostró valores discrepantes, caracterizando una muestra no normal de los resultados. Por esta razón, se optó por un análisis individualizado de los datos, considerando el nivel de valorización indicado por los entrenadores y entrenadores

$-7-$

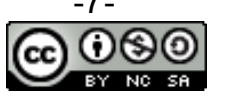

Esta obra está bajo una 
asistentes en cada ítem del instrumento, con posterior cálculo de la mediana general de los indicadores de acuerdo con los resultados de los entrenadores y entrenadores asistentes de forma separada. Los valores de mediana mayores o iguales a ocho (8.0) fueron adoptados para considerar los ítems más valorizados por los entrenadores y los entrenadores asistentes en relación a la procedencia de las fuentes de conocimiento. A pesar del bajo número de entrenadores en el estudio, se intentó identificar diferencias en la valorización de las FC entre los entrenadores y los entrenadores asistentes por medio de la prueba $U$ de Mann Whitney y comparar los resultados entre las comisiones técnicas (entrenador y entrenador asistente) de cada selección, por medio del test Kruskal Wallis (Leotti, Coster, y Riboldi, 2012). Los datos fueron analizados utilizando el software SPSS 23 (Statistics for Windows, Versión 23.0) y las figuras fueron construidas en las hojas electrónicas de cálculos en el software Office 2016.

\section{RESULTADOS}

Los ítems relacionados con las fuentes de conocimiento (FC) procedentes de la Experiencia Profesional (EP) y de la Formación Académica (FA) fueron más valorizados entre los entrenadores de las selecciones (Figura 1).

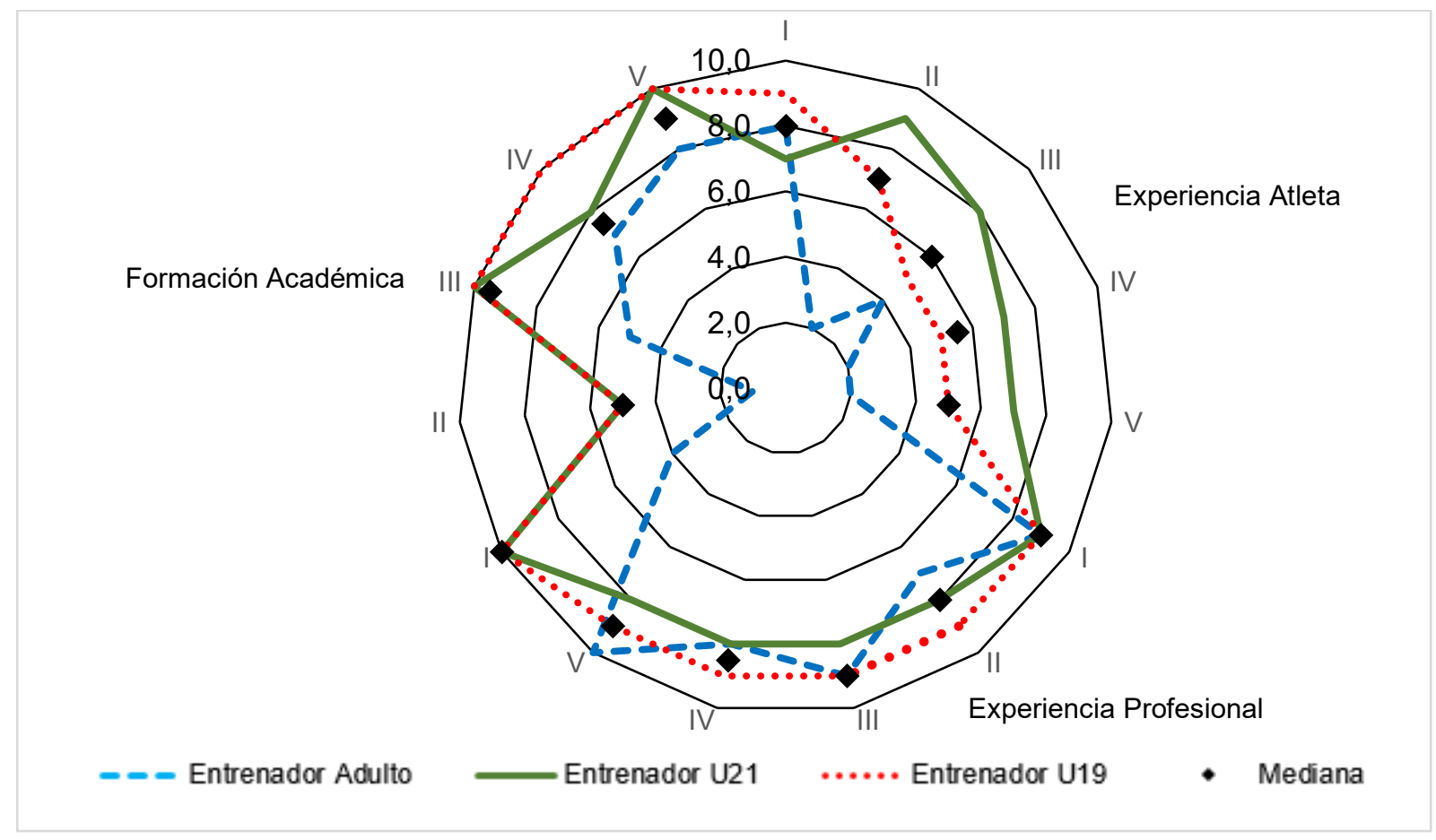

Figura 1. Mediana e indicadores de valorización en relación a la procedencia de las fuentes de conocimiento de los entrenadores de las selecciones U19, U21 y adulta. Nota: I, II, III, IV y $\mathrm{V}$ son los ítems de las tres dimensiones del instrumento. Fuente: Elaborado por los autores, 2020.

-8 - 
Sin embargo, el entrenador de la selección adulta valorizó más las FC procedentes de la EP, en especial los ítems relacionados con los aspectos metodológicos y conocimientos teóricos adquiridos por iniciativa propia y construidos de las experiencias profesionales, mientras que los entrenadores de las selecciones U19 y U21, además de las FC procedentes de la EP, también valorizaban las FC provenientes de la FA, como los ítems relacionados con los conocimientos y con los aspectos metodológicos adquiridos en los cursos de formación inicial en Educación Física y de formación y capacitación de entrenadores.

El mismo hecho ocurrió en los resultados de los entrenadores asistentes (Figura 2), con mayor valorización de los ítems relacionados a las FC procedentes de la EP y de la FA. En especial, a los ítems relacionados con aspectos metodológicos y conocimientos teóricos adquiridos por iniciativa propia, a partir de las experiencias profesionales y a los ítems relacionados con conocimientos y aspectos metodológicos adquiridos en cursos de formación y capacitación de entrenadores.

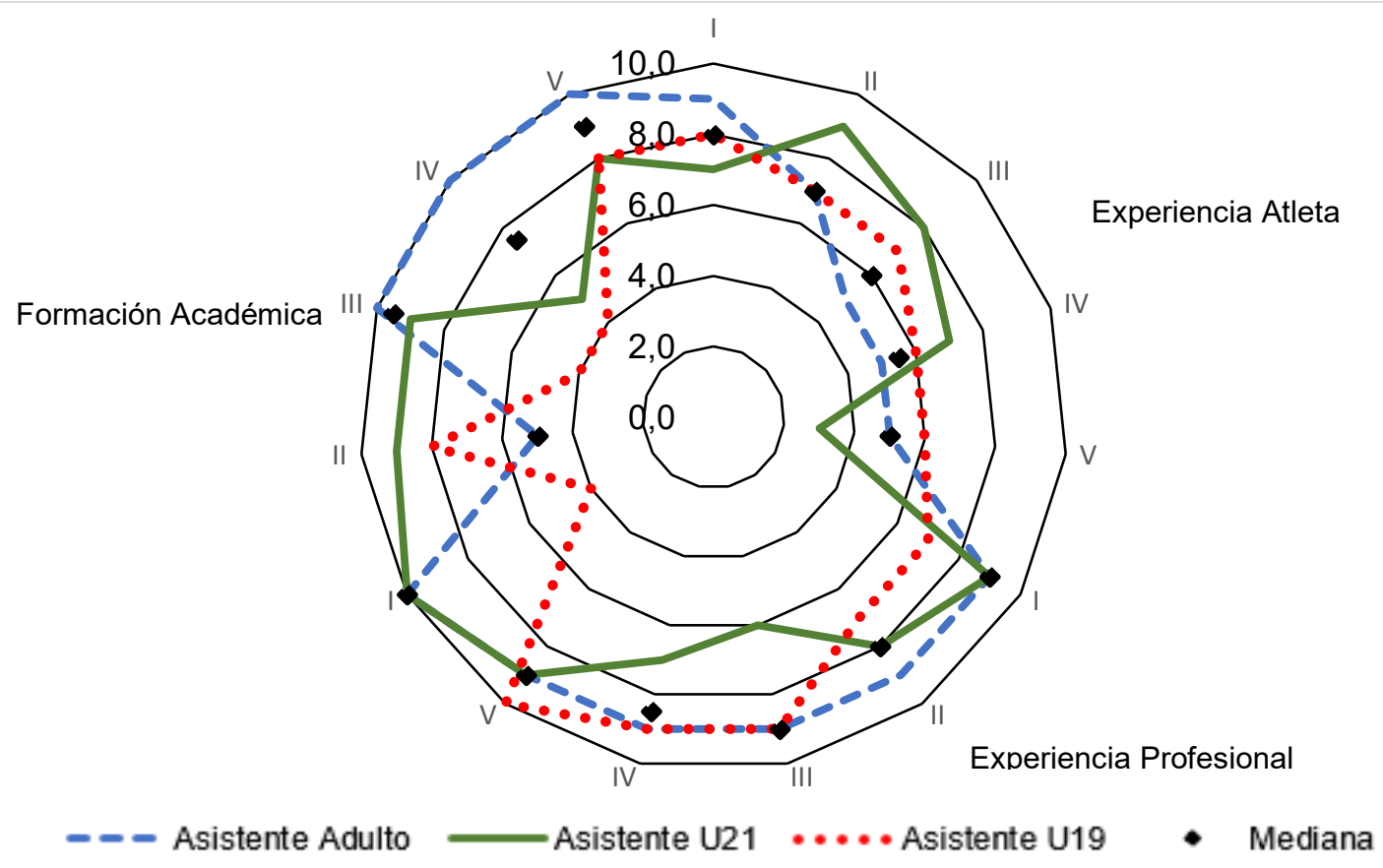

Figura 2. Mediana e indicadores de valorización de la procedencia de las fuentes de conocimiento de los entrenadores asistentes de las selecciones U19, U21 y adulta. Nota: I, II, III, IV y $\mathrm{V}$ son los ítems de las tres dimensiones del instrumento. Fuente: Elaborado por los autores, 2020.

Por otro lado, cuando se analizaron los resultados de las comisiones técnicas (entrenador y entrenador asistente) de cada selección (Figura 3), se observó que los ítems relacionados con las FC procedentes de la EP fueron más valorizados por los integrantes de las comisiones técnicas de las selecciones U19 (Me=9.0) y adulta $(\mathrm{Me}=9.0)$, mientras que los miembros de la comisión técnica de la selección U21 (Me=9.5) valorizaban más los ítems relacionados a las FC procedentes de la FA.

$-9$

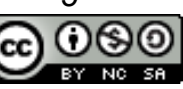

Esta obra está bajo una 
Sin embargo, no se detectaron diferencias significativas en los resultados del test de Kruskal Wallis entre las FC, independientemente de ser procedentes de la EP $\left(X^{2}=5.00 ; p>\right.$ $.05)$, FA $\left(X^{2}=.32 ; p>.05\right)$ o de la Experiencia del Atleta (EA) $\left(X^{2}=3.58 ; p>.05\right)$ entre las comisiones técnicas de las selecciones U19, U21 y adulta.

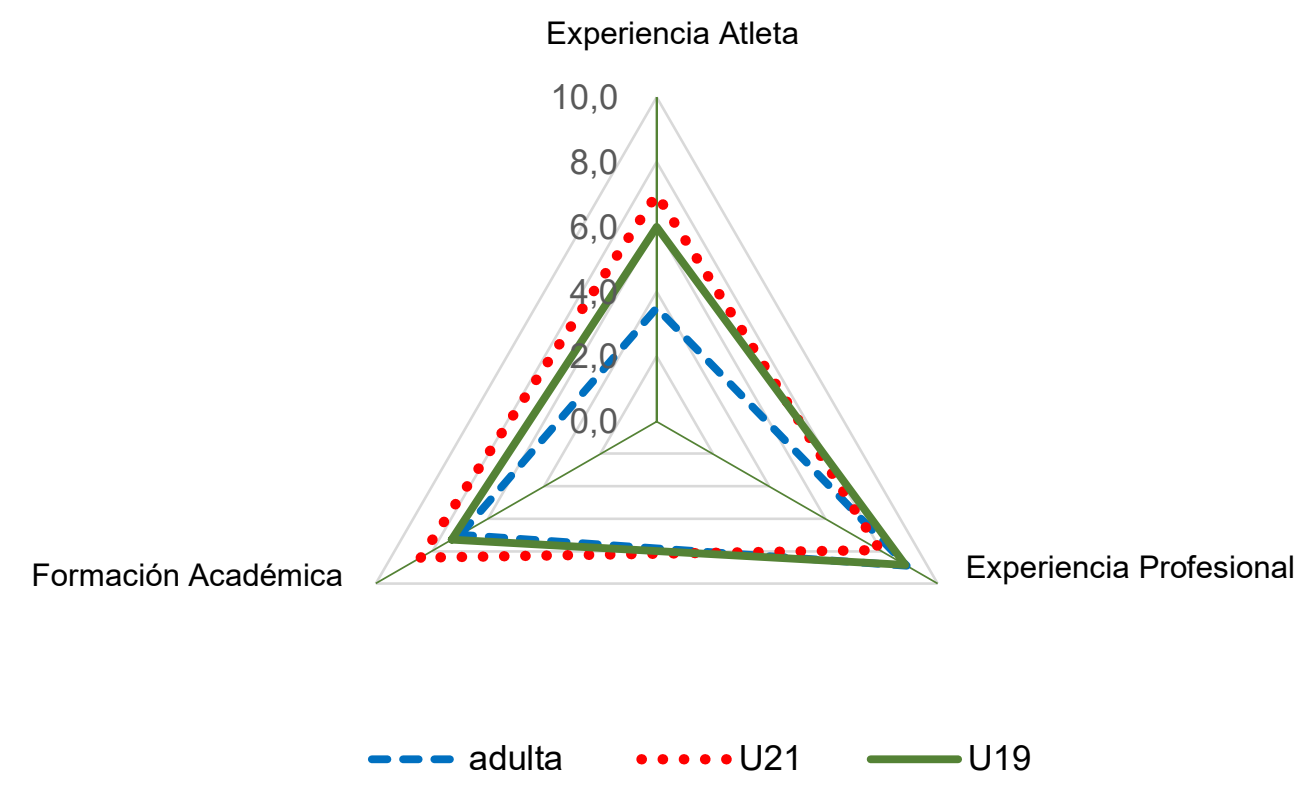

Figura 3. Mediana de la valorización de las FC de las comisiones técnicas de las selecciones U19, U21 y adulta. Fuente: Elaborado por los autores, 2020.

Entre otros resultados relevantes identificados, vale resaltar la baja valorización de los ítems relacionados con las FC procedentes de la experiencia de los atletas, por parte de los entrenadores y entrenadores asistentes de las selecciones U19, U21 y adulta, como también la ausencia de diferencias significativas en los resultados en la prueba $U$ de Mann Whitney en la valorización de los ítems de las FC procedentes de la EP $(U=4.50 ; p>.05)$, FA (U = 3.5; $p>.05)$ y $\mathrm{EA}(\mathrm{U}=2.50 ; p>.05)$, al comparar los resultados entre los entrenadores y los entrenadores asistentes de las selecciones U19, U21 y adulta.

\section{DISCUSIÓN}

A partir del objetivo de identificar las FC más valorizadas por los entrenadores y entrenadores asistentes en el ejercicio profesional y en la construcción del proceso de entrenamiento de las selecciones de balonmano masculino en las categorías U19, U21 y adulta, los resultados encontrados ratifican las ideas de algunos autores (Cunha et al., 2014; Cushion, Armour, y Jones, 2003), quienes afirman que las investigaciones con entrenadores adquieren características específicas del contexto regional, sin despreciar las vertientes individuales y sociales inherentes al proceso de entrenamiento deportivo. En el caso de Brasil, los aspectos relacionados a las exigencias legales de formación inicial en Educación Física para el ejercicio legal de la profesión de entrenador, han despertado el interés y la curiosidad 
de la comunidad científica internacional sobre la realidad brasileña (Milistetd, Trudel, Mesquita, y Nascimento, 2014).

En el caso de las selecciones brasileñas masculinas de balonmano, se constató una valorización similar entre las FC procedentes de la EP y FA y valorización superior de estas en relación a las $\mathrm{FC}$ procedentes de la EA. A pesar de que el estudio confirma los resultados de investigaciones internacionales, evidenciando la valorización de las FC procedentes de las experiencias de la práctica profesional (Cushion et al., 2003; Irwin, Hanton, y Kerwin, 2004; Stoszkowski y Collins, 2016; Wright et al., 2007), aún permanece una alta valorización de las FC procedentes de la "formación académica" (aprendizaje formal) por los entrenadores y entrenadores asistentes para el desempeño profesional y en la construcción del proceso de entrenamiento del modelo de juego de las selecciones masculinas de Balonmano.

Las exigencias legales de realización del curso de grado en Educación Física, como un requisito obligatorio para el desempeño profesional en la mayoría de las modalidades deportivas en Brasil, excepto para entrenadores de fútbol profesional y de algunas artes marciales/luchas, han suscitado una expectativa de que una formación en nivel superior sea traducida en mejor calificación y mayor reconocimiento social para la profesión de entrenador (ICCE, 2013). Sin embargo, los estudios han apuntado un bajo impacto de los cursos de graduación en la formación de los entrenadores deportivos en Brasil (Ramos et al., 2011; Rodrigues et al., 2017), aunque exista un robusto cuerpo de conocimientos profesionales desarrollado en estos cursos, la formación universitaria parece limitarse a preparar entrenadores para actuar, solamente, en el contexto de participación deportiva y proporciona pocas oportunidades diversas de experiencias de aprendizaje (Milistetd et al., 2014).

Además, la comunidad científica internacional ha hecho un llamado vehemente para la adhesión a programas de desarrollo profesional de entrenadores, en especial aquellos que integren situaciones de aprendizaje mediadas, no mediadas e internas (Werthner y Trudel, 2006), que estimulen una práctica reflexiva de los entrenadores (Gilbert y Trudel, 2001; Leggett y James, 2016; Nash y Sproule, 2012), capacitándolos para realizar las distintas adaptaciones en el complejo contexto de un proceso de entrenamiento.

Por otro lado, las FC procedentes de la EA fueron las menos valorizadas entre los entrenadores y entrenadores asistentes investigados, incluso con las elevadas experiencias atléticas competitivas internacionales vivenciadas por el entrenador de la selección U21 y el entrenador asistente de la selección U19 en Juegos Olímpicos y Campeonatos Mundiales. En este caso, los resultados pueden indicar que estas experiencias pueden estar aliadas a otras situaciones distintas del proceso de entrenamiento, como en la toma de decisiones en las relaciones interpersonales entrenador/entrenador asistente/atleta (Côté y Gilbert, 2009).

A pesar de que la experiencia atlética no es una condición necesaria para la formación de entrenadores de élite, hay un reconocimiento de que ella contiene elementos importantes en la adquisición de conocimientos para el ejercicio profesional (Mesquita, Isidro, y Rosado, 2010; Mesquita, Jones, Fonseca, y Silva, 2012). Por lo tanto, sería fundamental el incentivo de vivencias de situaciones distintas de aprendizaje en contextos informales en programas de formación y desarrollo de los entrenadores brasileños, como por ejemplo, la valorización de la experiencia como atleta, la experiencia práctica profesional, la participación en cursos de entrenadores, la supervisión de mentores y la interacción con otros entrenadores (Cushion et al., 2003; Irwin et al., 2004; Stoszkowski y Collins, 2016; Wright et al., 2007). 
Aunque no han sido observadas diferencias significativas en la comparación de las FC de entrenadores y entrenadores asistentes, se observó una mayor valorización de las FC procedentes de la EP, o sea, por medio de situaciones de aprendizaje informal. La permanencia del entrenador de nacionalidad española, con formación distinta a la de los entrenadores brasileños, en la dirección de la selección adulta masculina por casi una década y apuntando la participación en los Juegos Olímpicos de Pekín 2008 y Río 2016, parece haber afectado los demás entrenadores investigados. En este caso, la calificación y el reconocimiento internacional del entrenador de la selección adulta pudieron haber facilitado el acceso de los demás integrantes de las comisiones técnicas de las selecciones a experiencias de aprendizaje en contextos informales.

Las experiencias de aprendizaje en contextos informales como, por ejemplo, oportunidades para la interacción y observación de entrenadores de élite de esta modalidad, son reconocidas como FC de elevada contribución para el desarrollo de entrenadores de élite mundial (Erickson, Bruner, MacDonald, y Côté, 2008; Irwin et al., 2004; Rynne, 2012). De esta forma, el dinamismo y el elevado nivel de las exigencias competitivas internacionales, unidas a la experiencia acumulada en el desempeño profesional de del balonmano de élite, pueden ser consideradas continuas oportunidades de aprendizaje para los entrenadores y entrenadores asistentes de las selecciones brasileñas (Cushion et al., 2010; Hanratty y O'Connor, 2012; Occhino, Mallett, y Rynne, 2013; Rynne, Mallett, y Tinning, 2010).

Investigaciones similares con entrenadores de distintas modalidades en Brasil también revelaron una mayor valorización de las FC procedentes de la EP, con énfasis en los medios de aprendizaje informal (Ramos et al., 2011; Rodrigues et al., 2017), lo que parece ser un consenso en investigaciones internacionales con entrenadores de Balonmano (Cunha et al., 2014; Feu et al., 2012) y otras modalidades (González-Rivera et al., 2017; Mesquita et al., 2010).

Los resultados encontrados comprenden importantes subsidios para ser compartidos con la Confederación Brasileña de Balonmano, en el sentido de favorecer la implementación de estrategias de formación a largo plazo de los entrenadores, en especial aquellas que permitan cambios en las estructuras cognitivas de los entrenadores y fomenten la reflexión sobre la propia práctica profesional. A pesar de las limitaciones del estudio, principalmente con respecto a la utilización de, únicamente, datos de los entrenadores de las selecciones masculinas y en las temporadas deportivas de 2016 y 2017, las evidencias reflejan la naturaleza compleja del desarrollo profesional de los entrenadores de mayor expresión de la modalidad de balonmano en el país, pudiendo ser resultantes del desarrollo profesional de entrenadores deportivos en Brasil.

\section{CONCLUSIONES}

El desempeño profesional de los entrenadores y entrenadores asistentes de las selecciones brasileñas masculinas de las categorías U19, U21 y adulta de balonmano y la construcción del proceso de entreno del modelo de juego de las selecciones masculinas de balonmano se estructuran a partir de las FC procedentes de la "experiencia profesional". Sin embargo, aún existe una elevada valorización de las FC procedentes de la "formación académica", consideradas FC con menor influencia en el proceso de formación y desarrollo de los entrenadores de élite internacionales. 
La alta valorización del contexto formal de aprendizaje por los entrenadores investigados, en especial por los integrantes de la comisión técnica de la selección U21, puede estar relacionada a la sanción que propone la Ley N. 9696/98, donde se limita el desempeño profesional de entrenadores deportivos a aquellas personas que hayan obtenido el grado académico de Bachillerato en Educación Física, como también a las pocas oportunidades de situaciones de aprendizaje que superen a las perspectivas tradicionales de enseñanza adoptadas en los programas de desarrollo de entrenadores.

Los resultados encontrados indican la necesidad de ampliar las investigaciones que involucren los entrenadores de balonmano de Brasil en distintos niveles competitivos, como también en otras modalidades deportivas, en especial los estudios que puedan servir de soporte para el desempeño de los entrenadores en su realidad práctica. Además, el proceso de formación y desarrollo de los entrenadores a largo plazo debería ser una preocupación inmediata y constante de las entidades administrativas del deporte en el país.

TRADUCCIÓN AL ESPAÑOL: Ph.D. Elizabeth Carpio Rivera, Escuela de Educación Física y Deportes, Universidad de Costa Rica, Costa Rica.

\section{REFERENCIAS}

Amorim, A. M. (2017). Desenvolvimento tático-técnico no handebol masculino: estratégias utilizadas nos acampamentos nacionais promovidos pela Confederação Brasileira de Handebol (Tese de Doutoramento). Universidade Federal de Santa Catarina. Recuperado de https://repositorio.ufsc.br/handle/123456789/189453

Araújo, D., Teques, P., Hernández-Mendo, A., Reigal, R. E., y Anguera, M. T. (2016). La toma de decisión, ¿es una conducta observable?: Discusión sobre diferentes perspectivas teóricas utilizadas en el estudio del rendimiento deportivo. Cuadernos de Psicología del Deporte, 16(1), 183-196. Recuperado de https://revistas.um.es/cpd/article/view/254481

Borms, J. (2008). Directory of sport science: A journey through time: the changing face of ICSSPE (5a ed.). Ilinois, Estados Unidos: Human Kinetics.

Cañadas, M., Rodríguez, G., Feu, S., Parejo, I., y García, J. (2013). Relathionship between pedagogical content knowledge and coaching methods. Revista de psicologia del deporte, 22(1), 183-186. Recuperado de https://www.redalyc.org/pdf/2351/235127552017.pdf

Clemente, F., Martins, F. M., y Mendes, R. S. (2014). Periodization based on small-sided soccer games: Theoretical considerations. Strength \& Conditioning Journal, 36(5), 3443. doi: https://doi.org/10.1519/SSC.0000000000000067

Côté, J., y Gilbert, W. (2009). An integrative definition of coaching effectiveness and expertise. International Journal of Sports Science \& Coaching, 4(3), 307-323. doi: https://doi.org/10.1260\%2F174795409789623892

Cunha, A. F. V. P., Estriga, M. L. D., y Batista, P. M. F. (2014). Fontes de conhecimento percebidas pelos treinadores: estudo com treinadores de andebol da $1^{a}$ divisão de seniores masculinos em Portugal. Movimento: Revista de Educação Física da UFRGS, 20(3), 917-940. doi: https://doi.org/10.22456/1982-8918.43663 
Cushion, C., Armour, K., y Jones, R. (2003). Coach education and continuing professional development: Experience and learning to coach. Quest, 55(3), 215-230. doi: https://doi.org/10.1080/00336297.2003.10491800

Cushion, C., Nelson, L., Armour, K., Lyle, J., Jones, R., Sandford, R., y O'Callaghan, C. (2010). Coach learning and development: $A$ review of literature. London: The national Coaching Foundation.

Recuperado

de

https://www.researchgate.net/publication/265566741 Coach Learning and Developm ent A Review of Literature

Erickson, K., Bruner, M. W., MacDonald, D. J., y Côté, J. (2008). Gaining insight into actual and preferred sources of coaching knowledge. International Journal of Sports Science \& Coaching, 3(4), 527-538. doi: https://doi.org/10.1260\%2F174795408787186468

Falcão, W. R., Bennie, A., y Bloom, G. A. (2015). Desporto de alto rendimento: formação e competências do treinador. Em: Formação e Saberes em Desporto, Educação Física.

Feu, S. (2006). Organización didáctica del proceso de enseñanza-aprendizaje para la construcción del juego ofensivo en balonmano. E-Balonmano.com: Revista de Ciencias del Deporte, 2(4). Recuperado de http://www.ebalonmano.com/ojs/index.php/revista/article/view/12/10

Feu, S. (2018). El aprendizage del balonmano en la edad escolar desde el Modelo Tactical Game. Em S. Feu, J. García-Rubio, y S.J.G. Ibañez (Eds.), Avances cientificos para el aprendizage y desarrolo del Balonmano. Caceres, Brasil: Editora da UNEMAT.

Feu, S., Ibáñez, S. J., Calvo, A. L., Saiz, S. J., y Cañadas, M. (2012). El conocimiento profesional adquirido por el entrenador de balonmano: experiencias y formación. Revista de psicologia del deporte, 21(1), 107-115. Recuperado de https://bit.ly/3jugTiZ

Galatti, L., Bettega, O. B., Brasil, V. Z., de Souza Sobrinho, A. E. P., Bertram, R., Tozetto, A. V. B., ... Milistetd, M. (2016). Coaching in Brazil Sport Coaching as a Profession in Brazil: An Analysis of the Coaching Literature in Brazil From 2000-2015. International Sport Coaching Journal, 3(3), 316-331. doi: https://doi.org/10.1123/isci.2015-0071

Gilbert, W. D., y Trudel, P. (2001). Learning to coach through experience: Reflection in model youth sport coaches. Journal of teaching in physical education, 21(1), 16-34. Recuperado de https://bit.ly/3kMw48Z

González-Rivera, M. D., Campos-Izquierdo, A., Villalba, A. I., y Hall, N. D. (2017). Sources of knowledge used by Spanish coaches: A study according to competition level, gender and professional experience. International Journal of Sports Science \& Coaching, 12(2), 162-174. doi: https://doi.org/10.1177\%2F1747954117694733

Hanratty, M., y O'Connor, D. (2012). Understanding Expert Knowledge: A Case Study of Knowledge Acquisition in Elite Rugby League Strength and Conditioning Coaches. International Journal of Coaching Science, 6(1), 45-63.

Hill-Haas, S. V., Dawson, B., Impellizzeri, F. M., y Coutts, A. J. (2011). Physiology of smallsided games training in football: a systematic review. Sports Medicine, 41(3), 199-220. doi: https://doi.org/10.2165/11539740-000000000-00000

Ibáñez, S. J. (2008). La planificación y el control del entrenamiento técnico-táctico en baloncesto. En N. Terrados y J. Calleja (Eds.), Fisiología, entrenamiento y medicina del baloncesto (pp. 299-313). Barcelona: Paidotribo. 
Ibáñez, S. J., Mendoza, M. Á. P., y Calvarro, J. M. A. (1999). Taxonomía de medios para la iniciación al baloncesto. Red: revista de entrenamiento deportivo, 13(4), 15-24.

Ibáñez, S. J., Pérez-Goye, E., García-Rubio, J., y Courel-Ibáñez, J. (2019). Effects of task constraints on training workload in elite women's soccer. International Journal of Sports Science \& Coaching, 15(1), 90-107. doi: https://doi.org/10.1177/1747954119891158

International Council for Coaching Excellence (ICCE). (2013). International Sport Coaching Framework Version 1.2. Champaign, United States: Human Kinetics. Recuperado de https://www.icce.ws/ assets/files/iscf-1.2-10-7-15.pdf

Irwin, G., Hanton, S., y Kerwin, D. (2004). Reflective practice and the origins of elite coaching knowledge. Reflective Practice, 5(3), 425-442. doi: https://doi.org/10.1080/1462394042000270718

Jiménez, S., Lorenzo, A., y Gómez , M. Á. (2009). Medios de formación de los entrenadores expertos en baloncesto. Cultura Ciencia Deporte [CCD], 4(11), 119-126. Recuperado de https://dialnet.unirioja.es/servlet/articulo?codigo=3097154

Leggett, R., y James, J. (2016). Exploring the Benefits of a Coach Development Process ... on the Coach. International Journal of Human Resource Development: Practice, Policy \& Research, 1(2), 55-65. doi: https://doi.org/10.22324/ijhrdppr.1.116

Leotti, V. B., Coster, R., y Riboldi, J. (2012). Normalidade de variáveis: métodos de verificação e comparação de alguns testes não-paramétricos por simulação. Revista HCPA, 32(2), 227-234. Recuperado de https://seer.ufrgs.br/hcpa/article/view/29874/19186

Lyle, J., y Cushion, C. (Eds.). (2010). Sports Coaching: Professionalisation and Practice is a comprehensive evidence-based textbook of sports coaching theory and practice.

Mendes, J. C., Brandt, R., Carvalho, H. M., Feu, S., Ibañez, S. J., y Nascimento, J. V. (2019). Validation and reliability of a sources of knowledge scale for Brazilian coaches. Motriz: Revista de Educação Física, 25(3). doi: http://dx.doi.org/10.1590/S19806574201900030013

Menezes, R. P. (2018). Ensino do handebol em longo prazo: estudo a partir da opinião de treinadores. Educación Física y Ciencia, 20(2). Recuperado de http://sedici.unlp.edu.ar/handle/10915/70622

Menezes, R. P., Marques, R. F. R., y Nunomura, M. (2017). Teaching handball to players under-12: the perspective of Brazilian coaches. Motriz: Revista de Educação Física, 23(4), 1-8. doi: https://doi.org/10.1590/s1980-6574201700040006

Menezes, R. P., Ramos, N. C., Marques, R. F. R., y Nunomura, M. (2018). Teaching handball to U-16 and U-18 women's teams: coaches' perspective on the long-term. Motriz: Revista de Educação Física, 24(4). doi: http://dx.doi.org/10.1590/S19806574201800040001

Menezes, R. P., y Reis, H. H. B. (2014). Relação entre eficácia defensiva e elementos técnicotáticos do handebol a partir dos discursos de técnicos experientes. Journal of Physical Education, 25(4), 513-526. doi: https://doi.org/10.4025/reveducfis.v25i4.21709

Mesquita, I., Isidro, S., y Rosado, A. (2010). Portuguese coaches' perceptions of and preferences for knowledge sources related to their professional background. Journal of Sports Science \& Medicine, 9(3), 480-489. Recuperado de https://www.ncbi.nlm.nih.gov/pmc/articles/PMC3761720/ 
Mesquita, I., Jones, R., Fonseca, J., y Silva, L. D. M. (2012). Nova abordagem na formação de treinadores; O que mudou e porquê?. Em In construção da identidade profissional em educação física. A formação á intervenção (pp. 41-60).

Milistetd, M., Trudel, P., Mesquita, I., y Nascimento, J. V. (2014). Coaching and coach education in Brazil. International Sport Coaching Journal, 1(3), 165-172.

Montero, I., y León, O. G. (2007). A guide for naming research studies in Psychology. International Journal of Clinical and Health Psychology, 7(3), 847-862. Recuperado de https://www.redalyc.org/pdf/337/33770318.pdf

Nash, C., y Sproule, J. (2012). Coaches perceptions of their coach education experiences. International Journal of Sport Psychology, 43(1), 33-52. Recuperado de https://www.researchgate.net/publication/234092162 Coaches perceptions of their c oach education experiences

Nelson, L. J., Cushion, C. J., y Potrac, P. (2006). Formal, nonformal and informal coach learning: A holistic conceptualisation. International Journal of Sports Science \& Coaching, 1(3), 247-259. doi: https://doi.org/10.1260\%2F174795406778604627

Occhino, J., Mallett, C., y Rynne, S. (2013). Dynamic social networks in high performance football coaching. Physical Education and Sport Pedagogy, 18(1), 90-102. doi: https://doi.org/10.1080/17408989.2011.631003

Oliver, J. F. C. (2018). El balonmano deporte de organización compleja. El error un componente de la auto organización del juego: una propuesta de entrenamiento. Em S. Feu, J. García y S. J. Ibáñez (Coords.), Avances científicos para el aprendizaje y desarrollo del balonmano. Caceres, España: Universidad de Extremadura.

Ramos, V., Graça, A., Nascimento, J. V., y Silva, R. (2011). A aprendizagem profissional-as representações de treinadores desportivos de jovens: quatro estudos de caso. Motriz: Revista de Educação Física, 17(2), 280-291. doi: http://dx.doi.org/10.5016/19806574.2011v17n2p280

Rodrigues, H. A., Teixeira Costa, G. C., Dos Santos Junior, E. L., y Milistetd, M. (2017). As fontes de conhecimento dos treinadores de jovens atletas de basquetebol. Motrivivência, 29(51). doi: https://doi.org/10.5007/2175-8042.2017v29n51p100

Rynne, S. B. (2012). Performance coaching craft through work and study. Em P. Potrac, W. Gilbert y J. Denison (Eds.), Routledge handbook of sports coaching. Londres: Routledge.

Rynne, S. B., Mallett, C. J., y Tinning, R. (2010). Workplace learning of high performance sports coaches. Sport, Education and Society, 15(3), 315-330. doi: https://doi.org/10.1080/13573322.2010.493312

Stoszkowski, J., y Collins, D. (2016). Sources, topics and use of knowledge by coaches. Journal of Sports Sciences, 34(9), 794-802. doi: https://doi.org/10.1080/02640414.2015.1072279

Tarodo, J., Belmonte, M. J., Toro, E., y Ruano, M. Á. (2011). Opinión de los entrenadores sobre distribución de contenidos técnico-tácticos y pedagógicos en distintas categorías de baloncesto de formación. Cuadernos de Psicología del Deporte, 11(2), 51-62. Recuperado de https://www.redalyc.org/articulo.oa?id=227019296005

Travassos, B., Davids, K., Araújo, D., y Esteves, T. P. (2013). Performance analysis in team sports: Advances from an Ecological Dynamics approach. International Journal of 

Performance
Analysis
in Sport,
13(1),
83-95.
doi:

https://doi.org/10.1080/24748668.2013.11868633

Trudel, P., y Gilbert, W. (2006). Coaching and coach education. Handbook of physical education

(pp.

516-539).

London:

Sage.

doi:

https://doi.org/10.4135/9781848608009.n29

Werthner, P., y Trudel, P. (2006). A new theoretical perspective for understanding how coaches learn to coach. The Sport Psychologist, 20(2), 198-212. Recuperado de https://www.researchgate.net/publication/267936822 A New Theoretical Perspective for Understanding How Coaches Learn to Coach

Wright, T., Trudel, P., y Culver, D. (2007). Learning how to coach: the different learning situations reported by youth ice hockey coaches. Physical Education and Sport Pedagogy, 12(2), 127-144. doi: https://doi.org/10.1080/17408980701282019

Participación: A- Financiamiento, B- Diseño del estudio, C- Recolección de datos, D- Análisis estadístico e interpretación de resultados, E- Preparación del manuscrito. 